\title{
PÓS-FEMINISMO, PÓS-RACIALISMO E PÓS-COLONIALISMO: A COBERTURA MEDIÁTICA DA CAMPANHA DE JOACINE KATAR MOREIRA
}

\author{
Rita Basílio de Simões* \\ (D) https: / / orcid.org/0000-0001-6356-6042 \\ Inês Amaral ** \\ (D) https: / / orcid.org/ 0000-0003-4929-4866
}

\section{Resumo}

As relações entre género, poder e política ecoam de forma profunda na representação mediática de mulheres no campo político, normalizando modelos de feminilidade e conceções da participação no espaço público. Partindo deste pressuposto e recorrendo a uma abordagem interseccional ancorada numa perspetiva feminista, este artigo recorre à análise temática crítica para examinar a cobertura mediática da campanha para as eleições legislativas portuguesas de 2019 de Joacine Katar Moreira. Identificamos os discursos ideológicos prevalecentes, relacionando-os com os conceitos de pós-feminismo, pós-racialismo e pós-colonialismo e problematizando as suas implicações para o acesso igualitário à tomada de decisão política e para a efetiva promoção da igualdade de género.

Palavras-chave: Media informativos, mulheres políticas, representação, pós-feminismo, Joacine Katar Moreira.

\section{Abstract \\ Post-feminism, post-racialism and post-colonialism: The media coverage of Joacine Katar Moreira's campaign \\ The relations between gender, power and politics resonate deeply in the media representation of women in the political field, normalizing models of femininity and conceptions of participation in the public sphere. Based on this assumption and using an intersectional approach anchored to a feminist perspective, this article draws on critical thematic analysis to examine the media coverage of the campaign for the 2019 Portuguese legislative elections by Joacine Katar Moreira. We identify the prevailing ideological discourses, relating them to the concepts of post-feminism, post-racialism and post- colonialism, and questioning their implications for equal access to political decision-making and for the effective promotion of gender equality.}

Keywords: News media, political women, representation, post-feminism, Joacine Katar Moreira.

Univ Coimbra, Faculdade de Letras (FLUC), 3000-370 Coimbra, Portugal; Instituto de Comunicação da NOVA (ICNOVA), 1069-061 Lisboa, Portugal.

Endereço postal: Faculdade de Letras, Largo da Porta Férrea, 3000-370 Coimbra, Portugal.

Endereço eletrónico: rbasilio@fl.uc.pt

** Univ Coimbra, Faculdade de Letras (FLUC), 3000-370 Coimbra, Portugal; Centro de Estudos de Comunicação e Sociedade (CECS), Universidade do Minho, 4710-057 Braga, Portugal.

Endereço postal: Faculdade de Letras, Largo da Porta Férrea, 3000-370 Coimbra, Portugal.

Endereço eletrónico: ines.amaral@uc.pt 


\begin{abstract}
Resumen
Postfeminismo, postracialismo y poscolonialismo: la cobertura mediática de la campaña de Joacine Katar Moreira

Las relaciones entre género, poder y política resuenan profundamente en la representación mediática de las mujeres en el campo político, normalizando modelos de feminidad y concepciones de participación en la esfera pública. Basado en esta suposición y utilizando un enfoque interseccional anclado a una perspectiva feminista, este artículo se basa en un análisis temático crítico para examinar la cobertura mediática de la campaña para las elecciones legislativas portuguesas de 2019 por Joacine Katar Moreira. Identificamos los discursos ideológicos prevalecientes, relacionándolos con los conceptos de posfeminismo, posracialismo y poscolonialismo y cuestionando sus implicaciones para la igualdad de acceso a la toma de decisiones políticas y para la promoción efectiva de la igualdad de género.
\end{abstract}

Palabras clave: medios informativos, mujeres políticas, representación, postfeminismo, Joacine Katar Moreira.

\title{
Introdução
}

Iniciada em 22 de setembro de 2019, a campanha eleitoral para a XIV Legislatura Portuguesa ficou marcada pela circunstância de fazer aflorar no espaço público, sob intenso mediatismo, a primeira mulher negra no papel de cabeça de lista de um partido político em Portugal. A luso-guineense historiadora de profissão e desconhecida do grande público Joacine Elysees Katar Tavares Moreira (JKM) liderou a candidatura pelo círculo eleitoral de Lisboa do partido de Esquerda LIVRE, granjeando uma representação mediática que reproduz muitas das tensões conhecidas da relação entre género, poder e política, sem deixar de configurar um caso de estudo de representação política feminina singular.

A investigação da representação mediática de atores políticos tem mostrado como as mulheres tendem a figurar nos media informativos como um fenómeno novo e desviante relativamente às normas de género. Permanentemente julgadas perante o padrão normativo masculino, as mulheres candidatas, membros ou líderes de câmaras parlamentares ou executivos de Governo são, com frequência, reificadas como estranhas num campo globalmente dominado por homens, como mães e cuidadoras que estão fora do seu lugar e como mais cooperantes do que os atores políticos masculinos (Campus 2013). Todavia, a excecionalidade da figura de JKM, que acabou eleita deputada ao parlamento português, traduzida na cor da sua pele, na sua gaguez pronunciada, no seu ativismo feminista declarado, contaminou a cobertura mediática da figura política chave de um jovem partido fundado em 2013, apostado em conquistar representação parlamentar.

JKM configura, pois, um caso exemplar do escrutínio mediático cerrado de mulheres políticas, que está nos antípodas das dinâmicas clássicas de sub-representação. A investigação feminista dos media mostra que, originalmente, os meios de comunicação subestimam e menosprezam as candidatas políticas e mulheres 
parlamentares, sub-representando-as comparativamente com os políticos homens, negando-lhes títulos honoríficos, identificando-as pelo primeiro nome, por relações familiares com sujeitos masculinos ou usando descrições de género pouco lisonjeiras, sinalizando ao público que a política é, em última análise, «um jogo de homens» (Ross 2009). Nos últimos anos, outras formas de atuação mediática têm sido documentadas, nomeadamente a atenção exacerbada às figuras políticas femininas e às intersecções identitárias que personificam (Joshi, Hailu e Reising 2019), sujeitando esses atores sociais a uma «supervigilância» e hipervisibilidade na cobertura mediática (Ward 2017, 47).

Quando os media ignoram a relevância das identidades intersectoriais, tornam-nas invisíveis e esse efeito pode ser tão problemático para a promoção da igualdade de género quanto a sub-representação de mulheres relativamente aos homens (Gill 2007; Hayes e Lawless 2016; Haraldsson e Wängnerud 2019). Por outro lado, quando as identidades interseccionais são acentuadas, tal não conduz necessariamente a uma representação pública justa e não estereotipada e a uma afirmação dos direitos políticos das mulheres.

Partindo do pressuposto de que é também pelas representações mediáticas que o poder social flui (Simões 2017; Amaral et al. 2019), neste artigo, analisamos o modo como os principais media informativos portugueses representaram a candidata do LIVRE JKM durante o período de campanha eleitoral das últimas eleições legislativas portuguesas. A partir de uma abordagem interseccional ancorada numa perspetiva feminista, e recorrendo à análise temática crítica, identificamos os padrões de representação e os discursos ideológicos prevalecentes na cobertura mediática da candidata e problematizamos as suas implicações para o acesso igualitário à tomada de decisão política e para a real e efetiva promoção da igualdade de género na sociedade em geral.

\section{Media, género e política}

É consabido que persistem profundos desequilíbrios relativamente ao lugar e ao papel desempenhado por homens e mulheres nos media e na paisagem comunicacional em geral. Uma das preocupações que têm alimentado a investigação feminista em comunicação nas últimas décadas é justamente o modo como os media representam as mulheres políticas (Ross 2002), ainda que o número de mulheres na cena política tenha aumentado substancialmente um pouco por todo o mundo ocidental.

Globalmente, cinco grandes tendências têm sido identificadas pela investigação feminista das representações mediáticas de mulheres candidatas políticas no Ocidente. Uma dessas tendências é a da sua sub-representação. Independentemente do contexto geográfico, na paisagem mediática, as figuras políticas femininas tendem a ser significativamente menos visíveis do que os homens (Carlin e 
Winfrey 2009; Ross 2009; Martins 2015). Indissociável da histórica e global ausência de plenos direitos políticos, este padrão interliga-se com a difícil integração das mulheres no campo, não obstante a introdução, um pouco por todo o mundo, desde a década de 1990, de quotas eleitorais de género (Monteiro 2011). A sub-representação das mulheres na política e a persistência de padrões de comportamentos desfavoráveis à integração das preocupações das mulheres nos complexos parlamentários explicarão, assim, em parte, a razão pela qual, nas performances mediáticas, as mulheres continuam a figurar como estando fora do lugar (Campus 2013).

Claramente, estas são performances que reforçam o mesmo pensamento binário homem/mulher, homem no espaço público/mulher no espaço privado que estrutura outro dos padrões de representação de mulheres políticas, que se traduz não na invisibilidade, mas na visibilidade problemática, outra das faces do fenómeno que Gay Tuchman (1978) designou de aniquilação simbólica feminina. Com efeito, a investigação no campo documenta a tendência para uma cobertura centrada em assuntos relativamente triviais, tais como a aparência física, a forma de apresentação, o estado civil, a trajetória de infância e o estilo de vida e família, em lugar de preocupada com as ideias e propostas políticas (Braden 1996; Carlin e Winfrey 2009). Numa cobertura desta natureza, as mulheres políticas experimentam um intenso escrutínio das suas características pessoais, que pode contribuir para a sua objetificação (Wasburn e Wasburn 2011; Ritchie 2013), incluindo como objetos sexuais (Carlin e Winfrey 2009), e afetar a sua carreira política (Heflick e Goldenberg 2009; Haraldsson e Wängnerud 2019).

As performances mediáticas tendem, igualmente, a veicular uma cobertura mais negativa das mulheres comparativamente aos homens, com frequência questionando as suas características pessoais, a sua experiência e o conhecimento para levar a cabo uma liderança política eficaz (Devitt 1999; Carlin e Winfrey 2009). Ainda que alguns estudos mostrem que o impacto dos discursos estereotipados afeta de forma menos positiva os homens do que as mulheres (Fridkin, Kenney e Woodall 2009), a cobertura centrada nas particularidades individuais contribui para desqualificar as suas qualidades como sujeitos políticos, particularmente em áreas chave da vida social, tais como a economia e a segurança nacional (Carroll e Fox 2006; Devitt 1999).

Outra tendência da cobertura mediática é, justamente, a associação reiterada do perfil político feminino a questões consideradas «de mulheres», tais como a educação, a conciliação da vida profissional e familiar, a interrupção voluntária da gravidez, esvaziando, por vezes, a sua autoridade em matéria de questões ditas «masculinas» (Devitt 1999; Carroll e Fox 2006), mas contrariando a clássica noção de que as mulheres nem sempre parecem representar as mulheres quando adquirem poder político (Childs e Mona 2008).

Um quinto padrão da cobertura mediática de mulheres políticas mostra uma outra forma de atuação dos media informativos que, em lugar de ignorarem ou 
exacerbarem os atores políticos femininos como desviantes e fora do lugar, os normalizam no espaço dos órgãos decisórios. Embora reconheçam que as mulheres sempre enfrentaram experiências marcadamente sexistas, estes estudos sustentam que o cenário político mudou, que as mulheres deixaram de configurar novidades eleitorais, o que veio a repercutir-se na emergência de um leque mais diversificado de representações mediáticas, «normalizando» as mulheres na política (Hayes e Lawless 2016). Quando os media realçam, por exemplo, os contributos positivos de mulheres políticas na intervenção pública no domínio da igualdade de género, estão notoriamente a representar como virtuosas as mulheres que atuam no campo político (Joshi, Hailu e Reising 2019).

De facto, o maior acesso das mulheres à tomada de decisão pública, graças designadamente a opções, como a que em Portugal se tomou, de instituir limiares mínimos de paridade aplicáveis às listas de candidaturas apresentadas à Assembleia da República, ao Parlamento Europeu e aos órgãos eletivos das autarquias locais, favorecerá uma menor atratividade por uma cobertura centrada nas mulheres políticas por serem mulheres. Alguma investigação sugere mesmo que as mulheres políticas, não sendo já uma exceção, podem receber uma cobertura simpática e favorável, ao serem consideradas virtuosas em dimensões como a compaixão e a honestidade (Hayes e Lawless 2016) e mais motivadas ou capazes de promover a igualdade de género e a representação de grupos marginalizados (Verge e Pastor 2018).

Além das teses da novidade, da desqualificação e da normalização, a investigação feminista mais recente tem também destacado a tendência dos media para a identificação das identidades interseccionais (Cho, Crenshaw e McCall 2013). A partir da perspetiva da interseccionalidade, estes estudos têm sublinhado como as particularidades distintivas dos sujeitos políticos são iluminadas ou obscurecidas pelos media, com efeitos significativos na representação pública destes sujeitos (Ward 2017; Joshi, Hailu e Reising 2019). Cunhado por Crenshaw (1991), o conceito de interseccionalidade encerra uma teoria multidimensional que reconhece que as identidades sociais são dinâmicas, uma vez que se interseccionam em várias dimensões, social e culturalmente construídas, que desencadeiam processos de opressão, discriminação e desigualdades (Nogueira 2011).

Em última instância, o conceito de interseccionalidade questiona as interrelações de poder nas identidades sociais e estruturas (Crenshaw 1991) e favorece uma compreensão dos grupos identitários, ao permitir analisar as representações sociais e os processos de opressão que são moldados pelo género, raça, classe e particularidades pessoais distintivas associadas a desordens ou defeitos, a exemplo da gaguez. O plano dos valores e das ideologias é, por conseguinte, instrumental para pensar a construção mediática das identidades interseccionais das mulheres e a sua relação com o género, o poder e a política. 


\section{Pós-feminismo, pós-colonialismo, pós-racialismo}

O feminismo tornou-se mainstream (Fraser 2013) nos discursos públicos de empoderamento das mulheres que procuram criar uma ideia de um feminismo contemporâneo e popular (Banet-Weiser 2018a), que se traduz numa evidente ligação ao neoliberalismo (McRobbie 2004). Enquanto manifestação de feminismo popular, o pós-feminismo circula nos media, na Internet e na publicidade numa perspetiva mercantilizada, intensificando a ideia de um feminismo «cool», jovem e de self-empowerment (Banet-Weiser 2018a). Ancorado numa cultura neoliberal (Gill 2007), um feminismo individual e dirigido a mulheres brancas privilegiadas adquiriu particular popularidade nos media sociais e nas campanhas publicitárias (BanetWeiser e Portwood-Stacer 2017). Foi a este fenómeno suportado pelo chavão empreendedor do neoliberalismo que Zeisler (2016) apelidou de femvertising.

É no contexto desta subjetividade feminista que reside o pós-feminismo. Enquanto ideologia neoliberal, incorpora um foco individualista e enfatiza os discursos feministas liberais de independência, escolha e ação (Banet-Weiser 2018b). Assumindo uma disrupção com o questionamento das desigualdades estruturantes de género, a cultura neoliberal reifica os corpos femininos a performances hipersexualizadas (Álvares 2017) e normativas do que deve ser uma mulher (Amaral, Santos e Brites 2020). O constrangimento da escolha (McRobbie 2009) traduz-se também nas «fronteiras da feminilidade» (Álvares 2017, 103), o que reforça relações de poder ancoradas numa normatividade instrumentalizada pelo neoliberalismo na cultura popular (Banet-Weiser e Portwood-Stacer 2017; BanetWeiser 2018b) esvaziando os programas feministas da sua essência política (Vavrus 2002; Gill 2007). Este «commodity feminism» (Goldman 1992) alia o feminismo ao capitalismo da sociedade de consumo, criando a normalização de novas dinâmicas de sexismo e misoginia (Gill 2016; Simões e Silveirinha 2019). Dos usos simbólicos de género e da feminilidade (McRobbie 2009), o pós-feminismo apropria as velhas reivindicações dos movimentos feministas como forma de legitimação moral, visibilizando e despolitizando o feminismo (Vavrus 2002) e mercantilizando-o como um produto de cultura popular (Banet-Weiser 2018a).

A normatividade tem sido predominante nos discursos mediáticos e mediatizados, que incorporam também um espaço discursivo que afirma um feminismo «moderado» que normaliza as conquistas das mulheres. Enquadrada na ideologia pós-feminista, a «domesticação» é defendida por Dean (2010) como uma forma de incorporação do feminismo «moderado» nos media, por oposição ao feminismo «radical», que também reproduz mitos e estereótipos do sistema patriarcal.

A esta luz, as mulheres na política figuram, com frequência, representadas como um sujeito pós-feminista. Além de reificar uma ligação intrínseca entre a esfera pública e privada (Vavrus 2002, 131), o sujeito feminino pós-feminista também naturaliza a feminilidade branca com privilégios de classe, a marca distintiva das mulheres visíveis na política nacional e local (Carlin e Winfrey 2009). E é 
justamente por tornarem os elementos raciais e de classe invisíveis que estas dinâmicas comunicativas se articulam com as ideias do pós-racialismo, uma teoria que ganhou força com a eleição de Barack Obama para a presidência norte-americana, em 2008, num contexto em que o pós-colonialismo oblitera os contextos históricos e culturais das mulheres norte-americanas.

Os contextos sociais, históricos e culturais dos estudos pós-coloniais sobrepõem-se, frequentemente, a questões de género, raça e classe (Tyagi 2014). O pós-colonialismo celebra o anticolonialismo como ato de resistência, mas silencia as hierarquias de género em espaços racializados (Parashar 2016). A tensão entre os estudos pós-coloniais e a teoria feminista pós-colonial assenta na sub-representação das mulheres colonizadas. A teoria feminista pós-colonial mostra que a mulher colonizada sofre uma «dupla colonização» (Peterson e Rutherford 1986), na medida em que experimenta a opressão colonialista e patriarcal (Tyagi 2014).

A leitura das identidades subalternas a partir da epistemologia da alteridade resgata as experiências inviabilizadas das mulheres que foram reconstruídas como o «Outro» no pensamento moderno ocidental (Spivak 1989). A partir desta leitura, as mulheres colonizadas libertam-se das narrativas que as colocam como o «Outro» e são representadas no seu contexto histórico e cultural. O pensamento de Spivak (1989) assenta no questionamento dos instrumentos de poder e nas possibilidades de agência do sujeito subalterno no espaço pós-colonial genderizado. Criticando o silenciamento dos vários feminismos sobre este «Outro», Spivak (2010) aborda os «feminismos subalternos» por oposição a um feminismo universalista, eurocêntrico, ocidental e branco. As dinâmicas pós-coloniais alinham com o pós-feminismo e o pós-racialismo, reificando a opressão.

Os discursos do pós-racialismo assumem a retórica de uma espécie de «pós-direitos civis» (Kennedy 2017), relativizando a experiência do racismo (Lentin 2011). As ideias pós-racialistas assentam na perspetiva conservadora, numa interpretação livre da ciência, de que a raça não existe (Kennedy 2017), logo, o racismo não existe. As narrativas pós-raciais despolitizaram a questão racial e criaram um imaginário ancorado nos media e no entretenimento da indústria de Hollywood (Francisco 2017).

O pós-feminismo e o pós-racialismo assentam numa retórica neoliberal, projetando a ideia de que a consciência de género e raça são escolhas (Vavrus 2002; Kennedy 2017). Estes discursos hegemónicos ocultam estruturas de poder que perpetuam sistemas de opressão no que concerne a género, raça e classe. Nos media ecoam apropriações de campanhas feministas como «revolução», «poder» e «sobrevivência» numa reconfiguração do ativismo em estruturas corporativas que assumem a raça como o elemento que induz a ideia de diversidade e integração, aportando numa apropriação da luta antirracista. O pós-racialismo e o pós-feminismo popularizados nos media e na Internet, utilizando figuras como Oprah Winfrey e Hillary Clinton, perpetuam-se nas invisibilidades e sub-representações das mulheres fora do «padrão» normativo, discursos de alteridade e formas dissimuladas de discriminação. 


\section{A cobertura da campanha de JKM}

Reconhecendo a importância de produzir evidências empíricas quantitativas e qualitativas que permitam conhecer as performances mediáticas, a fim de apoiar a formação de consensos acerca da necessidade de intervenção nas políticas de comunicação dirigidas aos media, este artigo analisa a representação de JKM nos media mainstream portugueses. Tomando como horizonte teórico uma perspetiva feminista ancorada numa abordagem interseccional, analisamos a representação da candidata do LIVRE na produção informativa publicada nas plataformas online de quatro títulos da imprensa generalista portuguesa e de um jornal nativo digital durante a campanha para as eleições legislativas portuguesas de 2019, que decorreu de 22 de setembro e 4 de outubro, período chave do papel da mediação no debate de questões de interesse público e, ao mesmo tempo, da construção da imagem das figuras políticas.

Tradicionalmente, a análise da cobertura noticiosa da esfera política centra-se nos períodos de campanha eleitoral, tendência que pode explicar-se pelo elevado interesse pelo potencial impacto dos media junto do eleitorado. Com raízes no trabalho pioneiro de Lazarsfeld, nos Estados Unidos (Lazarsfeld et al. 1944), os estudos do noticiário político têm permitido uma compreensão mais profunda das relações que podem tecer-se entre o campo dos media e o campo da política e, em especial, da visibilidade pública das figuras políticas. A investigação empírica que tem procurado analisar diferentes momentos do ciclo eleitoral tem, além disso, distinguido as especificidades próprias dos períodos de campanha, entre elas o menor grau de personalização de atores políticos, em virtude de as performances mediáticas tenderem a centrar-se menos nos indivíduos do que nos partidos políticos que representam (Van Aelst e De Swert 2009; Vliegenthart, Boomgaarden e Boumans 2011). Em linha com estes estudos, privilegiámos uma análise do período de campanha eleitoral.

Três questões de investigação guiaram a análise. Concretamente: (1) Quais os principais padrões de representação mediática? (2) Como é que os padrões de representação predominantes se relacionam com as construções socioculturais de género, raça e classe e com o contexto sociocultural e ideológico mais amplo? (3) Quais os efeitos da cobertura mediática na campanha de JKM? Para responder a estas questões recorremos à análise temática crítica.

\section{Estratégia metodológica}

A análise temática tem sido descrita como uma abordagem descritiva qualitativa independente que oferece um método para «identificar, analisar e relatar padrões (temas)» nos dados em análise (Braun e Clarke 2006, 79). Neste artigo, lançámos mão de uma análise temática a partir de uma abordagem qualitativa 
crítica (Lawless e Chen 2019), recorrendo ao software de análise qualitativa MAXQDA. A análise temática crítica permite-nos examinar as inter-relações entre os discursos nos textos - no nosso caso, nos textos dos media informativos -, as práticas sociais, as relações de poder e as ideologias (Chen e Lawless 2016).

Relativamente ao objeto, dirigimos a atenção para a imprensa no atual ecossistema mediático. Partindo da consideração do papel nuclear que a imprensa sempre desempenhou na vida pública, estabelecendo, na proposta original de Lippmann (1922), «mapas conceptuais do mundo»e, já no quadro dos estudos do agenda-setting, reconduzíveis originalmente ao trabalho de McCombs e Shaw (1972), «fixando a agenda» de públicos e decisores políticos e dos outros media, centrámo-nos em cinco jornais. Trata-se de cinco meios de comunicação social com importantes índices de circulação em Portugal, que ilustram diferentes tendências editoriais e que representam bem o alcance do espectro político: os jornais diários Correio da Manhã, Diário de Notícias e Público, o semanário Expresso e o nativo digital Observador. Constituímos um corpus de análise recolhido das edições online dos quatro legacy media e do jornal digital durante as duas semanas de campanha eleitoral com peças que incidem diretamente sobre a candidata JKM. Reunimos 23 peças informativas, que agrupámos levando em conta o jornal de proveniência e o género jornalístico utilizado na sua construção, tal como mostra a Tabela 1.

Tabela 1.

Cobertura da campanha de JKM por meio de comunicação

\begin{tabular}{|c|c|c|c|}
\hline \multirow{2}{*}{ Jornal } & \multicolumn{3}{|c|}{$\mathbf{N}=\mathbf{2 3}$} \\
\hline & Notícia & Perfil & Reportagem \\
\hline Público & 1 & 1 & 0 \\
\hline Observador & 3 & 0 & 1 \\
\hline Expresso & 10 & 0 & 0 \\
\hline Diário de Notícias & 2 & 0 & 0 \\
\hline Correio da Manhã & 4 & 0 & 1 \\
\hline
\end{tabular}

Fonte: Elaboração própria

Recorrendo à análise temática crítica, e utilizando as ferramentas do software MAXQDA, procurámos observar os padrões discursivos dos textos informativos e as ideologias, posições de poder ou hierarquias que esses padrões mobilizam. O processo analítico desdobrou-se em duas fases, seguindo a proposta de Brandi Lawless e Yea-Wen Chen (2019). Primeiro, a partir de um processo de codificação aberta (Owen 1984), identificámos padrões textuais: repetições de palavras e de frases, recorrências de sentidos idênticos criados por palavras diferentes e elemen- 
tos discursivos realçados. Este processo permitiu-nos codificar os temas frequentes, proeminentes e vigorosos no corpus de análise. Os padrões identificados foram, numa segunda etapa, interligados com o contexto ideológico mais amplo e agrupados em macrotemas. O procedimento de definição destes macrotemas levou em conta, portanto, os elementos textuais observáveis, mas também os elementos sistematicamente mitigados ou invisíveis, que são tão ou mais relevantes para as práticas discursivas. Deste modo, interligámos os padrões textuais com a influência dos discursos sociais dominantes (Chen e Lawless 2018). As autoras coparticiparam ativamente na construção dos temas e macrotemas.

A Figura 1 dá conta do resultado final deste exercício. Perpassam pelo corpus sete temas principais, que articulámos em dois macrotemas nucleares, que aglutinam o essencial da normatividade subjacente à cobertura mediática de JKM.

\section{Figura 1.}

Temas e macrotemas da cobertura mediática de JKM durante a campanha eleitoral para as eleições legislativas portuguesas de 2019

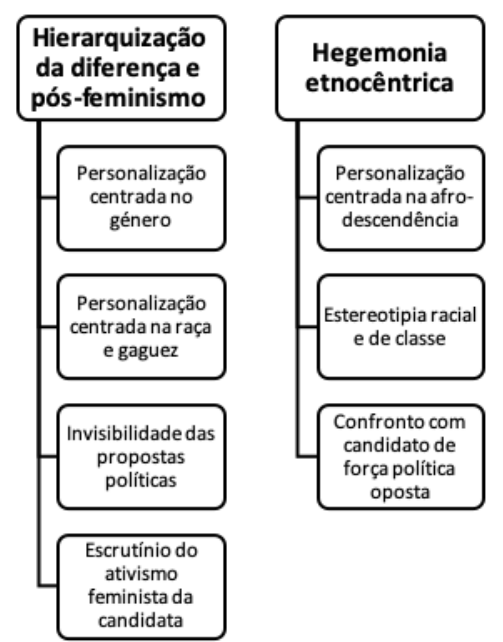

Fonte: Elaboração própria

\section{Análise e discussão de resultados}

«Hierarquização da diferença e pós-feminismo» é o macro tema com maior expressão no corpus de análise e que está intimamente relacionado com a excecionalidade dos atributos de JKM, que lhe outorga um valor-notícia, isto é, um interesse como matéria-prima suscetível de ser transformada em notícia (Wolf, 1987) muitíssimo elevado. 
Uma ativista negra a caminho do Parlamento? (Público, 4-10-2019)

O título interrogativo publicado pelo jornal Público no final da campanha ilustra bem como raça e género se articulam no discurso público, justificando uma cobertura orientada para a novidade da situação. Uma novidade deveras apetecível para os media mainstream. A exacerbação destas categorias - mulher e negra parece à superfície posicionar a candidata num lugar de desafio e de resistência dupla aos padrões do espaço político, considerado eminentemente masculino e branco. Contudo, tal como a investigação feminista também tem mostrado, a cobertura de excecionalidade, da novidade, tende a reificar o padrão.

Por outro lado, a visibilização do «Outro» por oposição aos padrões normativos dominantes não significa necessariamente uma dinâmica discursiva emancipadora, particularmente quando assenta, como é o caso, no efeito de "personalização» típico da política contemporânea, em que os atores se tornaram mais importantes do que as identidades coletivas dos partidos que representam (McAllister 2007). Contudo, a personalização pode ser altamente problemática. Com efeito, o foco reiterado na sua gaguez, bem como o questionamento persistente do modo como pode afetar a sua atuação política, configura menos uma força de inclusão do que de fomento do escrutínio das competências da candidata.

Na verdade, várias campanhas de desinformação e de difamação online dirigidas a JKM foram gizadas recorrendo justamente à exploração das suas características pessoais. O escrutínio da sua integridade como sujeito político começou desde logo a propósito do questionamento, veiculado nos media informativos, da genuinidade da sua gaguez. O que a limitada investigação da cobertura mediática da deficiência tem realçado é a existência de diferentes modelos de representação problemática, entre eles o modelo de patologia social (Clogston apud Haller 1993), à luz do qual as pessoas portadoras de deficiência são desfavorecidas e carecem do apoio do Estado, um apoio que é menos um direito do que uma dádiva. Outros modelos, a exemplo do modelo de consumo (Haller 1993), que destaca as suas qualidades como nicho de mercado inexplorado, não configuram necessariamente uma representação emancipadora. Neste caso, a gaguez de JKM aparece publicamente representada como falsa ou exagerada para granjear mediatismo e votos.

Depois de a gaguez ter ocupado parte do discurso sobre a candidata, surgiram nos últimos dias acusações de que Joacine não seria gaga, mas estaria a usar o facto como arma de campanha. (Expresso, 4-10-2019)

Bem se compreende que, perante um cenário dominado pela personalização baseada no género, raça e gaguez, as propostas políticas de JKM sejam absolutamente periféricas, além do que esta personalização ignora o papel de académica. JKM emerge no espaço público não como uma intelectual, não como historiadora ou especialista, mas, sim, como ativista feminista e dos direitos humanos. E esse 
ativismo, quando visível, também é sujeito a escrutínio, ou por ser considerado limitado ou extemporâneo:

A campanha da Joacine e do Livre tem insistido muito nos direitos das minorias e das mulheres que não são assim tão minoria - antes pelo contrário. (Observador , 2-10-2019)

Se olharmos para este tipo de discursos como exemplos de enunciados que mostram como foram supostamente resolvidas as reivindicações feministas «do passado» e garantidas as condições de igualdade, podemos interpretá-los como discursos construídos a partir de uma retórica neoliberal pós-feminista (Gill 2007), que não apenas desqualificam a agenda da candidata, como também a desqualificam a si.

Relativamente ao segundo conjunto de temas, «Hegemonia etnocêntrica», um primeiro aspeto que deve realçar-se é que, na cobertura mediática, JKM não é somente mulher, negra e gaga: é afrodescendente, condição que é sempre exaustivamente explorada, colocando em evidência e hiper-visibilizando a sua origem africana:

Apresentar como cabeça de lista por Lisboa uma mulher negra, afrodescendente e gaga é, assume Joacine Katar Moreira, «um incentivo para que qualquer indivíduo sinta que está habilitado a participar politicamente.» (Expresso, 24-9-2019)

A visibilidade conseguida é um êxito notável para Joacine, de 37 anos, negra nascida na Guiné-Bissau, a viver em Portugal desde os oito. (Correio da Manhã, 25-9-2019)

Este padrão discursivo centrado na personalização da sua afrodescendência pode ser visto como uma forma de reificar relações de hegemonia etnocêntrica e, em última instância, de uma ideia de colonialidade acomodada na sinalização da excecionalidade. Trata-se, pois, igualmente, de uma força cultural e ideológica que, pelo discurso, (re)produz e propaga ideologias dominantes (Hall 1997).

O recurso, ainda que com menos frequência, à estereotipia racial, que repousa no negro pobre, oriundo de bairros periféricos, a quem é oferecida uma oportunidade de ouro de derrubar barreiras, é uma outra forma de normalizar a condição minoritária e desfavorecida da população afrodescendente:

O historiador, eurodeputado entre 2009 e 2014 [Rui Tavares], vê em Joacine um exemplo de «genuinidade» e de como «é possível as pessoas comuns fazerem parte da política.» (Expresso, 28-9-2019)

De realçar também que, tal como sucede no excerto acima indicado, JKM é, com frequência, Joacine, diferentemente de Rui Tavares, fundador do partido LIVRE, que é nomeado de forma formal e identificado pela sua especialidade académica. 
Um outro tema visível na cobertura mediática da candidata JKM que se articula com uma força hegemónica etnocêntrica é o seu posicionamento em confronto sistemático com o candidato de uma força política oposta, André Ventura, do partido nacionalista CHEGA!

A candidata do Livre, comparando com o Presidente do Brasil, considerou que «Bolsonaro é um exemplo muito semelhante ao do André Ventura aqui.» (Correio da Manhã, 4-10-2019)

Este confronto da mulher afrodescendente de Esquerda com o português branco de Direita, instigado não raro pelo modo como as entrevistas jornalísticas são conduzidas, é expectável. É expectável, porque o confronto político, a altercação, a dicotomia entre extremos, são apetecíveis do ponto de vista mediático. Podemos, por outro lado, entender este padrão discursivo como um reflexo da campanha da candidata, com o objetivo de desviar a atenção da sua experiência política limitada, tal como foi sugerido relativamente à cobertura de algumas mulheres políticas, a exemplo de Sarah Palin (Wasburn e Wasburn 2011). Mas o recurso reiterado a esta fórmula é problemático e tem implicações na relação entre género, poder e política e, de modo mais amplo, na igualdade de género em geral.

\section{Conclusão}

A análise da cobertura mediática da candidata às eleições legislativas JKM mostra, à superfície, uma construção que contraria a invisibilidade das mulheres políticas nos media, posicionando-a num lugar de desafio e de resistência aos padrões normativos do espaço político, tradicionalmente masculino e branco. Contudo, a personalização reiterada e a hipervisibilidade do seu perfil singular traduzem-se numa «supervigilância» (Ward 2017) que, não tendo afetado de forma negativa os resultados eleitorais, se repercutiram, claramente, na imagem e carreira política de JKM e, sugerimos, no próprio imaginário da condição feminina no espaço público e político.

A cobertura dos primeiros meses de atividade parlamentar da deputada fez emergir no espaço público uma torrente de outros conflitos, nomeadamente com o também recém-eleito deputado André Ventura e com o seu próprio partido. JKM acusou o LIVRE de ter usado a sua condição de mulher negra para obter subvenções. ${ }^{1}$

«Joacine: 'Não podem exigir que deixe de gaguejar de uma hora para outra'», Jornal de Notícias online, 5-11-2020, URL: https:/ / www.jn.pt/nacional/joacine-nao-podem-exigir-que-deixe-de-gaguejar-de-uma-hora-para-outra-11482574.html; «Assembleia do Livre retira confiança política a Joacine Katar Moreira», Correio da Manhã online, 31-1-2020, URL: https:/ / www.cmjornal.pt/poli tica/ detalhe/ assembleia-do-livre-retira-confianca-a-joacine-katar-moreira; «André Ventura 'propõe' que Joacine 'seja devolvida ao seu país de origem'. Livre queixa-se de racismo,» Observador, 28-1- 
O partido retirou a confiança política à parlamentar que, a 3 de fevereiro de 2020, adquiriu no Parlamento o estatuto de deputada não inscrita. ${ }^{2}$

Por outro lado, é expectável que a cobertura estereotipada de novidade que documentámos, ainda que possa funcionar como uma mola propulsora para desviar a atenção de um perfil com experiência política limitada, eventualmente usado de forma instrumental pela candidata e/ou pelo seu partido, não favorece a normalização das mulheres no campo político e nas diferentes esferas de poder decisório.

Paralelamente, os media mainstream que examinámos reproduziram uma sensibilidade pós-feminista do mundo e reificaram uma hegemonia etnocêntrica, que mergulha as suas raízes num passado recente pouco discutido, porém disseminado nas práticas discursivas e culturais.

Considerando o alcance limitado do estudo que conduzimos e a visibilidade problemática da agora deputada JKM, sugerimos que, a partir igualmente de uma abordagem feminista qualitativa, estudos futuros analisem a cobertura da atividade parlamentar de mulheres políticas portuguesas, incidindo sobre diferentes tipos de meios de comunicação e levando em conta a importância da articulação das suas qualidades identitárias, desde logo de género, raça e classe, e os ambientes políticos e mediáticos em que se movem.

\section{Referências bibliográficas}

Álvares, Cláudia. 2017. «Pós-feminismo, misoginia online e a despolitização do privado.» Media E Jornalismo 17(30): 99-110.

Amaral, Inês et al. 2019. «(In)visibilities of Men and Aging in the Media: Discourses from Germany and Portugal». Lecture Notes in Computer Science vol. 11593: 20-32. DOI: https: / / doi.org/10.1007 / 978-3-030-22015-0_2

Amaral, Inês, Sofia José Santos e Maria José Brites. 2020. «Mapping Intergenerational Masculinities on Instagram». Lecture Notes in Computer Science vol. 12209: 3-16. DOI: https: / / doi.org/10.1007 /978-3-030-50232-4_1

Banet-Weiser, Sarah. 2018a. Empowered: Popular Feminism and Popular Misogyny in an Economy of Visibility. Durham, NC: Duke University Press.

Banet-Weiser, Sarah. 2018b. «Postfeminism and Popular Feminism.» Feminist Media Histories 4(2): 152-156. DOI: https:/ / doi.org/10.1525/ fmh.2018.4.2.152

Banet-Weiser, Sarah e Laura Portwood-Stacer. 2017. «The traffic in feminism: An introduction to the commentary and criticism on popular feminism.» Feminist Media Studies 17(5): 884-888. DOI: https:/ / doi.org/10.1080/14680777.2017.1350517

-2020, URL: https: / / observador.pt/2020/01/28/andre-ventura-propoe-que-joacine-seja-devolvidaao-seu-pais-de-origem-livre-queixa-se-de-racismo/.

«Joacine deixa de representar o Livre e passa a deputada não inscrita a partir de hoje,» Público online, 3-2-2020, https:/ / www.publico.pt/2020/02/03/politica/noticia/joacine-deixa-representar-livre-passa-deputada-naoinscrita-partir-hoje-1902643 
Braden, Maria. 1996. Women Politicians and the Media. Lexington, KY: University Press of Kentucky.

Braun, Virginia e Victoria Clarke. 2006. «Using thematic analysis in psychology.» Qualitative Research in Psychology 3(2): 77-101. DOI: https:/ / doi.org/10.1191/1478088706qp063oa

Campus, Donatella. 2013. Women Political Leaders and the Media. Basingstoke: Palgrave Macmillan.

Carlin, Diana B. e Kelly L. Winfrey. 2009. «Have you come a long way, baby? Hillary Clinton, Sarah Palin, and Sexism in 2008 Campaign Coverage.» Communication Studies 60(4): 326-343. DOI: https: / / doi.org/10.1080/10510970903109904

Carroll, Susan J. e Richard L. Fox 2006. Gender and Elections: Shaping the Future of American Politics. Cambridge: Cambridge University Press.

Chen, Yea-Wen e Brandi Lawless. 2018. «'Oh my god! You have become so Americanized': Paradoxes of adaptation and strategic ambiguity among female immigrant faculty.» Journal of International and Intercultural Communication 11(1): 1-20. DOI: https:/ / doi. org/10.1080/17513057.2017.1385825

Chen, Yea-Wen e Brandi Lawless. 2016. «Immigrant Women Negotiating Shifting Meanings of Work and Confronting Micro-aggressions with/in the Ivory Tower.» In Immigrant Workers and Meanings of Work: Communicating Life and Career Transitions, organizado por Suchitrae Shenoy-Packer e Elena Gabor, 99-111. New York: Peter Lang.

Childs, Sarah e Lena Krook Mona. 2008. «Critical mass theory and women's political representation.»Political Studies 56(3): 725-736. DOI: https://doi.org/10.1111/j.1467-92 48.2007.00712.x

Cho, Sumi, Kimberlé Williams Crenshaw e Leslie McCall. 2013. «Toward a Field of Intersectionality Studies: Theory, Applications, and Praxis.» Signs: Journal of Women in Culture and Society 38(4): 785-810.

Crenshaw, Kimberle. 1991. «Mapping the Margins: Intersectionality, Identity Politics, and Violence Against Women of Color». Stanford Law Review 43, 1241-1299.

Dean, Jonathan. 2010. Rethinking Contemporary Feminist Politics. Basingstoke: Palgrave Macmillan.

Devitt, James. 1999. Framing Gender on the Campaign Trail: Women's Executive Leadership and the Press. Washington, DC: Women's Leadership Fund.

Francisco, Flávio. 2017. «A utopia pós-racial nos Estados Unidos: reestruturação do racismo e a ascensão de Barack Obama na era do colorblindness.» Revista de História da UEG 6(1): 01-23.

Fraser, Nancy. 2013. Fortunes of Feminism: From State-Managed Capitalism to Neoliberal Crisis. London and New York: Verso Books.

Fridkin, Kim L., Patrick J. Kenney, e Gina Serignese Woodall. 2009. «Bad for men, better for women: The impact of stereotypes during negative campaigns.» Political Behavior 31(1): 301-324.

Gill, Rosalind. 2007. «Postfeminist media culture: Elements of a sensibility.» European Journal of Cultural Studies 10(2): 147-166. DOI: https: / / doi.org/10.1177/1367549407075898

Gill, Rosalind. 2016. «Post-postfeminism?: New feminist visibilities in postfeminist times.» Feminist Media Studies 16(4): 610-630.

Goldman, Robert. 1992. Reading Ads Socially. London and New York: Routledge.

Hall, Stuart. 1997. Representation: Cultural Representations and Signifying Practices. London: Sage.

Haller, Beth. 1993. «Paternalism and Protest: Coverage of Deaf Persons in the Washington Post and the New York Times.» Comunicação apresentada à $75^{\circ}{ }^{\circ}$ Annual Meeting of 
the Association for Education in Journalism and Mass Communication (Montreal, Quebec, Canadá, 5-8 de agosto, de 1992). URL: https://files.eric.ed.gov/fulltext/ ED351698.pdf

Haraldsson, Amanda e Lena Wängnerud. 2018. «The Effect of Media Sexism on Women's Political Ambition: Evidence from a Worldwide Study.» Feminist Media Studies 19(4): 525-41. DOI: https: / / doi.org/10.1080/14680777.2018.1468797

Hayes, Danny e Jennifer L. Lawless. 2016. Women on the Run: Gender, Media, and Political Campaigns in a Polarized Era. Cambridge: Cambridge University Press.

Heflick, Nathan A. e Jamie L. Goldenberg. 2009. «Objectifying Sarah Palin: Evidence That Objectification Causes Women to Be Perceived as Less Competent and Less Fully Human.» Journal of Experimental Social Psychology 45(3): 598-601. DOI: https:/ / doi. org / 10.1016/j.jesp.2009.02.008

Joshi, Devin K., Meseret F. Hailu e Lauren J. Reising. 2019. «Violators, Virtuous, or Victims? How Global Newspapers Represent the Female Member of Parliament.» Feminist Media Studies, 1-21. DOI: https: / / 10.1080/14680777.2019.1642225

Kennedy, Tanya Ann. 2017. Historicizing Post-Discourses: Postfeminism and Postracialism in United States Culture. New York: SUNY Press.

Lazarsfeld, Paul, Bernard Berelson e Hazel Gaudet. 1944. The People's Choice: How the Voter Makes up his Mind in a Presidential Campaign. New York: Columbia University Press.

Lawless, Brandi e Yea-Wen Chen. 2019. «Developing a method of critical thematic analysis for qualitative communication inquiry.» Howard Journal of Communications 30(1): 92-106. DOI: https: / / doi.org/10.1080/10646175.2018.1439423

Lentin, Alana. 2011. «What Happens to Anti-Racism When We Are Post Race?» Feminist Legal Studies 19(2): 159-168. DOI: https: / /10.1007/s10691-011-9174-5

Lippmann, Walter. 1922. Public Opinion. New York: Macmillan.

Martins, Carla. 2015. Mulheres, Liderança Politica e Media. Lisboa: Alethêia.

McAllister, Ian. 2007. «The Personalization of Politics.» In The Oxford Handbook of Political Behavior, organizado por Russell Dalton e Hans-Dieter Klingemann, 571-588. New York: Oxford University Press.

McCombs, Maxwell E. e Donald L. Shaw. 1972. «The Agenda-Setting Function of Mass Media.» The Public Opinion Quarterly 36(2): 176-187.

McRobbie, Angela. 2004. «Post-feminism and Popular Culture.» Feminist Media Studies 4(3): 255-264. DOI: https: / / doi.org/10.1080/1468077042000309937

McRobbie, Angela. 2009. The Aftermath of Feminism: Gender, Culture and Social Change. London: Sage.

Monteiro, Rosa. 2011. «A Política de Quotas em Portugal: O papel dos partidos políticos e do feminismo de Estado». Revista Crítica de Ciências Sociais 99: 31-50. DOI: https: / / doi. org / $10.4000 /$ rccs.3953

Nogueira, Conceição. 2011. «Introdução à Teoria da Interseccionalidade nos Estudos de Género.» In Género e Ciências Sociais, organizado por Sofia Neves, 67-78. Maia: Edições ISMAI.

Owen, William Foster. 1984. «Interpretive themes in relational communication.» Quarterly Journal of Speech 70(3): 274-287.

Parashar, Swati. 2016. «Feminism and Postcolonialism: (En)gendering Encounters.» Postcolonial Studies 19(4): 371-77. DOI: https: / / doi.org/10.1080/13688790.2016.1317388

Petersen, Kirsten H. e Anna Rutherford. 1986. A Double Colonization: Colonial and Post-colonial Women's Writing. Mundelstrup, Denmark: Dangoroo Press. 
Ritchie, Jessica. 2013. «Creating a monster: Online media constructions of Hillary Clinton during the Democratic Primary Campaign, 2007-8». Feminist Media Studies 13(1): 102-119. DOI: https: / / doi.org/10.1080/14680777.2011.647973

Ross, Karen. 2002. Women, Politics, Media: Uneasy Relations in Comparative Perspective. Cresskill, NJ: Hampton Press.

Ross, Karen. 2009. Gendered Media: Women, Men and Identity Politics. Lanham, MD: Rowman \& Littlefield.

Simões, Rita Basílio. 2017. «Do escrutínio dos media aos media sob escrutínio: estereótipos de género no espaço público mediatizado.» In Pessoas e ideias em trânsito: percursos e imaginários, organizado por Rita Basílio de Simões et al., 13-35. Coimbra: Imprensa da Universidade de Coimbra.

Simões, Rita Basílio e Maria João Silveirinha. 2019. «Framing street harassment: Legal developments and popular misogyny in social media.» Feminist Media Studies 1-17. DOI: https:/ / doi.org/10.1080/14680777.2019.1704816

Spivak, Gayatri Chakravorty. 1989. «Who Claims Alterity?» In Remaking History: Discussions in Contemporary Culture, organizado por Barbara Kruger e Phil Mariani, 269-292. Seattle: Bay Press.

Spivak, Gayatri Chakravorty. 2010. Pode o subalterno falar? Novo Horizonte: Editora UFMG. Tuchman, Gay. 1978. Making News: A Study in the Construction of Reality. New York: Free Press. Tyagi, Ritu. 2014. «Understanding Postcolonial Feminism in relation with Postcolonial and Feminist Theories.» International Journal of Language and Linguistics 1(2): 45-50.

Van Aelst, Peter e Knut De Swert. 2009. «Politics in the News: Do Campaigns Matter? A Comparison of Political News During Election Periods and Routine Periods in Flanders (Belgium).» Communications 34(2): 149-68.

Vavrus, Mary Douglas. 2002. Postfeminist News: Political Women in Media Culture. Albany: State University of New York Press.

Verge, Tània e Raquel Pastor. 2018. «Women's Political Firsts and Symbolic Representation.» Journal of Women, Politics \& Policy 39(1): 26-50. DOI: https:/ / doi.org/10.1080/15544 77X.2016.1268878

Vliegenthart, Rens, Hajo G. Boomgaarden, e Jelle W. Boumans. «Changes in political news coverage: Personalization, conflict and negativity in British and Dutch newspapers.» In Political communication in postmodern democracy, organizado por Kees Brants e Katrin Voltmer, 92-110. London: Palgrave Macmillan.

Ward, Orlanda. 2017. «Intersectionality and Press Coverage of Political Campaigns: Representations of Black, Asian, and Minority Ethnic Female Candidates at the UK 2010 General Election.» International Journal of Press/Politics 22(1): 43-66. DOI: http:/ / doi.org/10.1177/1940161216673195.

Wasburn, Philo C. e Mara H. Wasburn. 2011. «Media coverage of women in politics: The curious case of Sarah Palin.» Media, Culture E Society 33(7): 1027-1041. DOI: https:/ / doi.org/10.1177/0163443711415744

Wolf, Mauro. 1987. Teorias da Comunicação. Lisboa: Editorial Presença.

Zeisler, Andi. 2016. We Were Feminists Once: From Riot Grrrl to CoverGirl, the Buying and Selling of a Political Movement. New York: Public Affairs. 
Rita Basílio de Simões. Professora Auxiliar da Faculdade de Letras da Universidade de Coimbra. Investigadora integrada do ICNOVA e investigadora associada do Centro de Estudos Sociais da Universidade de Coimbra, os seus interesses de investigação têm cruzado os estudos do jornalismo e dos media digitais e a investigação feminista em comunicação. Membro da ECREA e da APEM, coordena atualmente o Grupo de Trabalho em Género e Sexualidades da Associação Portuguesa de Ciências da Comunicação.

Endereço eletrónico: rbasilio@fl.uc.pt

Inês Amaral. Professora Associada da Faculdade de Letras da Universidade de Coimbra. Doutorada em Ciências da Comunicação pela Universidade do Minho, é investigadora integrada do Centro de Estudos de Comunicação e Sociedade e investigadora associada do Centro de Estudos Sociais da Universidade de Coimbra. Tem investigado sobre sociabilidades nas redes sociais digitais, literacia mediática e digital, tecnologias e envelhecimento ativo, audiências e consumos mediáticos na era digital.

Endereço eletrónico: ines.amaral@uc.pt

Artigo recebido em 19 de julho e aceite para publicação, após revisão, em 7 de outubro de 2020. 